\title{
Acceleration of Polarized Protons in the AGS with Two Helical Partial Snakes
}

\author{
H. Huang, L.A. Ahrens, M. Bai, K. Brown, E.D. Courant \\ C. Gardner, J.W. Glenn, R.C. Gupta, A.U. Luccio \\ W.W. MacKay, V. Ptitsyn, T. Roser, S. Tepikian, N. Tsoupas
}

E. Willen, A. Zelenski, K. Zeno
Presented at the Particle Accelerator Conference (PAC'05) Knoxville, Tennessee
May 16-20, 2005

Collider-Accelerator Department

\author{
Brookhaven National Laboratory \\ F.O. Box 5000 \\ Upton, NY 11973-5000 \\ www.bnl.gov \\ Managed by \\ Brookhaven Science Associates, LLC
}

for the United States Department of Energy under

Contract No. DE-AC02-98CH10886 


\title{
DISCLAIMER
}

This report was prepared as an account of work sponsored by an agency of the United States Government. Neither the United States Government nor any agency thereof, nor any of their employees, nor any of their contractors, subcontractors, or their employees, makes any warranty, express or implied, or assumes any legal liability or responsibility for the accuracy, completeness, or any third party's use or the results of such use of any information, apparatus, product, or process disclosed, or represents that its use would not infringe privately owned rights. Reference herein to any specific commercial product, process, or service by trade name, trademark, manufacturer, or otherwise, does not necessarily constitute or imply its endorsement, recommendation, or favoring by the United States Government or any agency thereof or its contractors or subcontractors. The views and opinions of authors expressed herein do not necessarily state or reflect those of the United States Government or any agency thereof.

FOR UNCLASSIFIED, UNLIMTTED STI PRODUCTS

Available electronically at:

OSTI:

http://www.osti.gov/bridge

Available for a processing fee to U.S. Department of Energy and its contractors, in paper from:

\author{
U.S. Department of Energy \\ Office of Scientific and Technical Information \\ P.0. Box 62 \\ Oak Ridge, TN 37831 \\ Phone: (865) 576-8401 \\ Facsimile: (865) 576-5728 \\ E-mail: reports@adonis.osti.gov
}

National Technical Information Service (NTIS):

Available for sale to the public from:

U.S. Department of Commerce

National Technical Information Service

5285 Port Royal Road

Springfield, VA 2.2131

Phone: (800) 553-6847

Facsimile: (703) 605-6900

Online ordering: hattp://www.ntis.gov/ordering.htm 


\title{
ACCELERATION OF POLARIZED PROTONS IN THE AGS WITH TWO HELICAL PARTIAL SNAKES*
}

\author{
H. Huang, L.A. Ahrens, M. Bai, K. Brown, E. D. Courant, C. Gardner, J.W. Glenn, R. C. Gupta, \\ A.U. Luccio, W.W. MacKay, V. Ptitsyn, T. Roser, S. Tepikian, N. Tsoupas, E. Willen, A. Zelenski, \\ K. Zeno, BNL, Upton, USA
}

\author{
M. Okamura, J. Takano, Radiation Laboratory, RIKEN, Saitama, Japan
}

F. Lin, Indiana University, Bloomington, IN 47405

\begin{abstract}
The RHIC spin program requires $2 \times 10^{11}$ proton/bunch with $70 \%$ polarization. As the injector to RHIC, AGS is the bottleneck for preserving polarization: there is no space for a full snake to overcome numerous depolarizing resonances. An ac dipole and a partial snake have been used to preserve beam polarization in the past few years. Two helical snakes have been built and installed in the AGS. With careful setup of optics at injection and along the ramp, this combination can eliminate all depolarizing resonances encountered during acceleration. This paper presents the setup and preliminary results.
\end{abstract}

\section{INTRODUCTION}

Acceleration of polarized proton beams in a circular synchrotron is complicated by the numerous depolarizing spin resonances. There are mainly two kinds of resonances: imperfection resonances due to magnet field errors and misalignments, and intrinsic resonances due to betatron motion in quadrupoles. The imperfection resonance happens when $\mathrm{G} \gamma=\mathbf{n}$, where $\mathbf{n}$ is an integer, $\mathrm{G}=(\mathrm{g}-2) / 2=1.7928$ is the anomalous factor of the magnetic moment and $\gamma$ is the Lorentz factor. G $\gamma$ gives the number of full spin precessions for every orbit revolution and is also called the spin tune $v_{\mathrm{sp}}$.

The intrinsic resonance strength is proportional to the square root of beam energy. The intrinsic resonance happens when $v_{\mathrm{sp}}=\mathrm{G} \gamma=\mathrm{mP} \pm v$, where $\mathrm{m}$ is an integer, $\mathrm{P}$ is the super-periodicity of the synchrotron and $v$ is the betatron tune. In general, the intrinsic resonance is only associated with the vertical betatron tune $v_{\mathrm{y}}$. However, when coupling between the two transverse planes is present, the resonance could also occur at the location of the horizontal betatron tune: $\mathrm{G} \gamma=\mathrm{mP} \pm v_{\mathrm{x}}$. The so-called coupling resonance strength is proportional to the coupling and the strength of the original resonance[1]. In the Brookhaven Alternating Gradient Synchrotron (AGS),
$\mathrm{P}=12$.

When passing through a depolarizing resonance with constant crossing speed $\alpha$, the ratio of final polarization $\mathrm{P}_{-} \mathrm{f}$ to initial polarization $\mathrm{P}_{-} \mathrm{i}$ is given by the FroissartStora formula[2]:

$$
\frac{P_{f}}{P_{i}}=2 \exp \left(-\pi|\varepsilon|^{2} /(2 \alpha)\right)-1 .
$$

where $\varepsilon$ is the resonance strength and $\alpha=d(G \gamma) / d \theta . \theta$ is the orbit angle in the synchrotron. For intrinsic resonances and a beam with Gaussian transverse distribution:

$$
\frac{P_{f}}{P_{i}}=\frac{1-\pi|\varepsilon|^{2} / \alpha}{1+\pi|\varepsilon|^{2} / \alpha}
$$

where $\varepsilon$ is the spin resonance strength for the particle with the rms amplitude. For the AGS acceleration rate of $\alpha=$ $5 \times 10^{-5}$ a resonance strength of 0.004 leads to complete depolarization in either case. Typical resonance strengths in the AGS for a beam with a normalized $95 \%$ emittance of $10 \pi \mathrm{mm}$ mrad are between 0.0001 and 0.01 and therefore most of the resonances cause significant depolarization.

Over the past ten years, a $5 \%$ partial solenoidal Siberian Snake has been used successfully to overcome imperfection resonances in the AGS[3]. In addition, a coherent spin resonance excited by an ac dipole was used to overcome the four strong intrinsic spin resonances [4]: $\mathrm{G} \gamma=0+v_{\mathrm{y}}, 12+v_{\mathrm{y}}, 36 \pm v_{\mathrm{y}}$. The three weak ones at $\mathrm{G} y=24 \pm v_{\mathrm{y}}, 48-v_{\mathrm{y}}$ were left uncorrected. The ac dipole generated a full spin flip without significant emittance growth. Due to the linear coupling induced by the solenoidal field of the partial snake, there exist the socalled coupling depolarizing resonances which are driven by vertical motion with horizontal betatron frequency. The two betatron tunes have to be well separated to reduce the coupling effect but can not eliminate it completely. 


\section{STRONG PARTIAL SIBERIAN SNAKE}

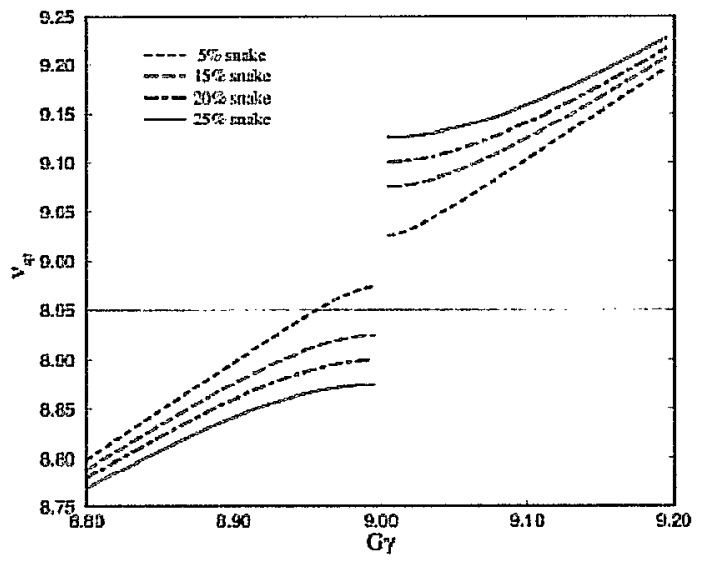

Figure1. Spin tune for various partial Siberian snake strength. The straight line indicates a possible value for vertical betatron tune.

However, the ac dipole technique only works for strong intrinsic resonances, since it relies on the strength of the intrinsic resonances to induce a strong enough artificial resonance. In a medium energy synchrotron such as AGS, there also exist intrinsic resonances which are not strong enough to be overcome by an ac dipole but cause some depolarization with the normal resonance crossing rate. As can be seen from Fig. 1, with a strong enough partial snake, the spin tune gap can be increased to allow placing the betatron tune inside the gap so that the intrinsic resonance conditions (both strong and weak) can be avoided. The challenge is to run the synchrotron with tune close to an integer.

A partial Siberian snake can be constructed by a solenoid or dipoles. A solenoid magnet causes significant coupling between the two transverse planes, while a dipole snake causes a large orbit excursion, especially at low energies. It is not practical to use a dipole partial snake in the AGS energy range. A helical snake design[5] can reduce the orbit excursion but orbit matching around the helical snake is still a problem. It is the double pitch design that solved the problem and made it possible to use a helical dipole snake with less coupling in the AGS.

\section{TWO PARTIAL SIBERIAN SNAKES}

With a partial Siberian snake the stable spin direction reverses direction at all imperfection resonances but is very close to the vertical direction at half-integer values of $\mathrm{G} \gamma$ as long as the partial snake is relatively weak. It is therefore possible to inject and extract vertically polarized beam at these energy values without much loss of polarization. The AGS injection and extraction energies are set to occur at $G \gamma=4.5$ and 46.5 , respectively.

For a strong partial snake, however, polarization loss at injection and extraction is no longer negligible. A $20 \%$
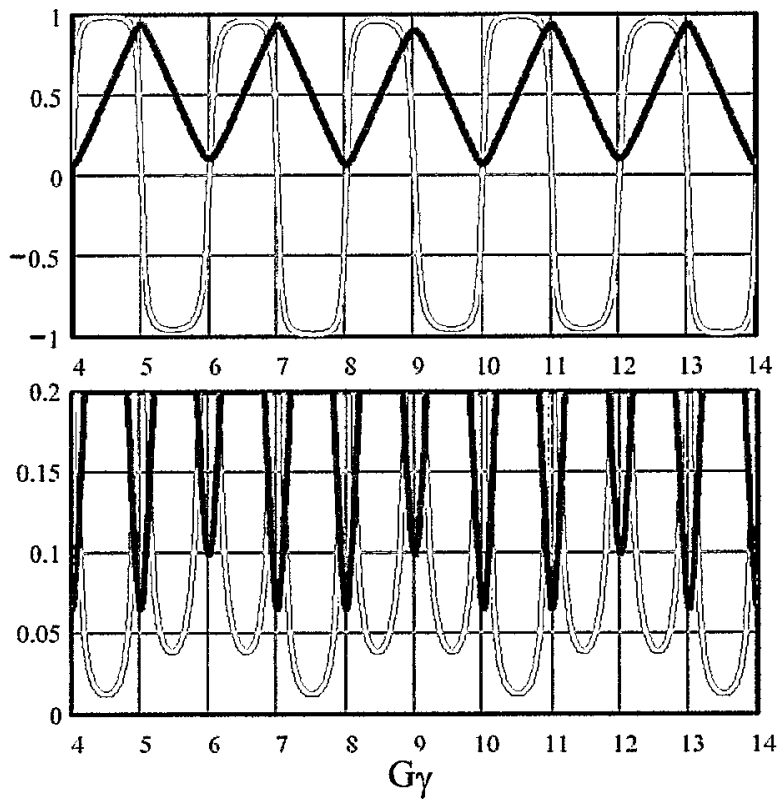

Figure 2: Top figure shows spin tune (blue, dark) and vertical component of the stable spin direction (red, light) for a $5 \%$ and $15 \%$ partial Siberian snake separated by $120^{\circ}$ orbital deflection angle as a function of $\mathrm{G} \gamma$. The bottom figure shows the deviation from an integer for the same quantities.

( $36^{\circ}$ spin rotation) snake will lead to a $10 \%$ polarization loss due to this spin direction mismatch. This could be solved with appropriate spin rotators in the injection and extraction beam lines. However, a single additional partial snake located in the AGS can provide the spin direction matching at injection and extraction and also increase the effective partial snake strength if its position is chosen properly. The location and the precession axis direction of multiple partial Siberian snakes has to be chosen very carefully to maintain control of the spin tune in a similar way as is necessary for multiple full Siberian snakes. For practical partial Siberian snakes the precession axis direction is always very close to longitudinal, which leaves only the location and strength of the partial snakes as free parameters.

Separating the two partial snakes by one third of the ring is of particular interest since it will introduce a periodicity of three units in the spin tune dependence on G $\gamma$. Since both the super-periodicity of the AGS (12) and the vertical betatron tune $(\sim 9)$ are divisible by three the spin tune will be the same at all intrinsic resonances, namely for $\mathrm{G} \gamma=3 \mathrm{n}$. With both snakes at equal strength, the effective snake strength doubles at $\mathrm{G} \gamma=3 \mathrm{n}$. At the injection and extraction energies, for which $G \gamma=3 n+1.5$, the two snakes cancel. The polarization direction in the AGS is therefore exactly vertical and no polarization is lost due to spin direction mismatch.

The $6 \%$ warm partial snake[6] has been installed in 2004 and used with an ac dipole to provide polarized proton beams for RHIC operation since last year. The 


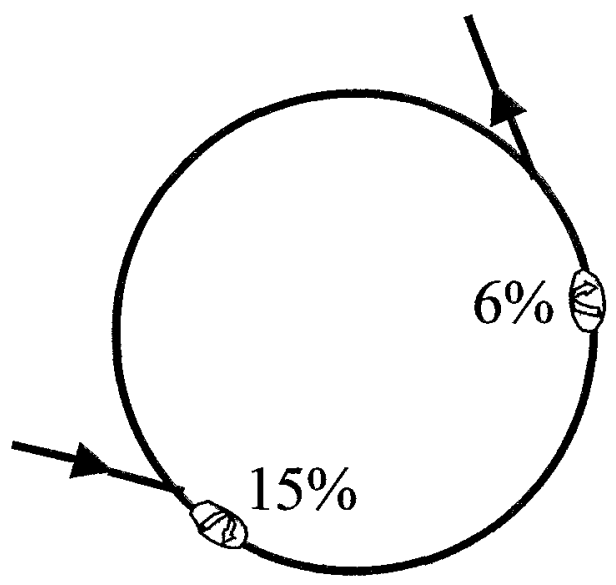

Figure 3: Locations of the partial Siberian snakes and the injection and extraction regions that give minimum polarization loss due to spin direction mismatch.

cold partial snake[7] has been installed this spring and is being commissioned. The cold snake is capable of being a $20 \%$ partial snake. Since spin matching at extraction and injection is much better with two properly arranged partial snakes, we are going to run the two snakes together. The advantage is obvious: a very substantial reduction of the injection and extraction spin mismatch can be achieved; at the same time the effective strength of the partial snakes at the intrinsic resonances is significantly increased. Fig. 2 shows the spin tune and the vertical component of the spin direction in the AGS with two partial snakes with rotation angles of $9^{\circ}(5 \%$ partial snake) and $27^{\circ}$ (15\% partial snake), respectively. The injection and extraction regions have to be located as shown in Fig. 3 relative to the location of partial Siberian snakes. In this case the polarization loss due to injection and extraction mismatch is only $2.4 \%$.

\section{HELICAL SNAKE OPERATION}

In the AGS, the polarized proton beam passes through 42 imperfection resonances and 7 intrinsic resonances. The warm helical snake magnet was run in the ring at $2680 \mathrm{~A}$ with constant field. It is an $8 \%$ and $6 \%$ partial snake at injection and extraction, respectively. The lattice distortion from the warm partial snake was manageable. Polarization at AGS extraction was $50 \%$ for an intensity of $10^{11}$ per bunch with warm helical snake--- a factor of 1.3 gain from the solenoid snake case. Currently this beam is used for RHIC operations. The remaining polarization loss has several sources: the limited aperture at $0+v_{y}$ prevents ac dipole to exercise its full power; the three weak intrinsic resonances at $24 \pm v_{\mathrm{y}}$ and $48-v_{\mathrm{y}}$ which are too weak to be overcome by an ac dipole.

The cold snake causes significant orbit distortion, especially at low energies. The betatron tune can not be set near an integer at injection. Instead, the plan is to ramp vertical betatron tune from nominal tune 8.70 up to 8.96 at $\mathrm{G} y=7$, before the resonance $0+v_{\mathrm{y}}$. Special quadrupole and dipole correctors are added outside the snake to correct tunes and orbit. In the foreseen difficulty of setup AGS with $15 \%$ cold snake, an intermediate $5 \%$ cold snake is first commissioned at injection field without an energy ramp. This way, the lattice with all correction magnets on but just cold snake off is still a stable lattice and easy to compare with model predictions. Polarization will be measured with the $5 \%$ cold snake on to confirm it affects spin as expected. The next step is to commission the cold snake at $15 \%$, then setup the ramp with vertical tune properly maintained near an integer. Polarization will then be measured after the first intrinsic resonance $0+v_{y}$. The energy ramp up to normal AGS extraction energy $24.3 \mathrm{GeV}$ will follow.

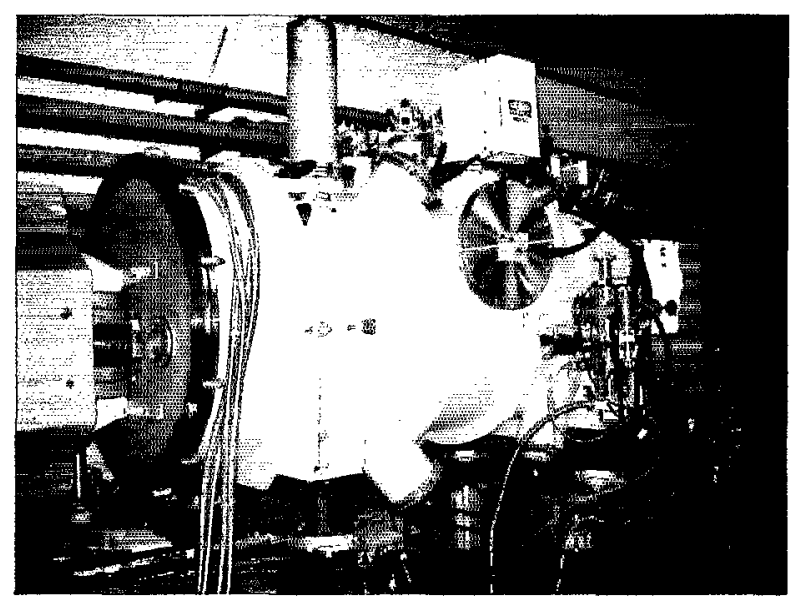

Figure 4: The cold snake installed in the AGS ring. Beam is going from left to the right in this picture.

\section{CONCLUSIONS}

$50 \%$ polarization at AGS extraction has been achieved with an ac dipole and a $6 \%$ helical partial snake. A strong $15 \%$ helical partial snake is being commissioned, which should provide $70 \%$ polarization required by RHIC. In general, the new partial Siberian snake design using helical dipoles with varying pitch make it possible to avoid depolarization from both imperfection and intrinsic spin resonances in medium energy accelerators and also maintain good matching to the vertical polarization in the injection and extraction beam lines.

\section{REFERENCES}

[1] H. Huang, T. Roser, A. Luccio, Proc. of 1997 PAC, Vancouver, May, 1997, p.2538.

[2] M. Froissart and R. Stora, Nucl. Instrum. Meth. 7, 297(1960).

[3] H. Huang, et al., Phys. Rev. Lett. 73, 2982 (1994).

[4] M. Bai, et al., Phys. Rev. Lett. 80, 4673 (1998).

[5]V.I. Ptitsyn and Yu.M. Shatunov, Nucl. Instrum. Meth. A398, (1997) 126.

[6] J. Takano, et al., these proceedings.

[7] E. Willen, et al., these proceedings. 